\title{
Die Viskosität und ihre Bedeutung für die Chemie des Zelluloids in Theorie und Praxis.
}

Von H. Schwarz (Aboshi, Japan).

(Eingeg. am 28. Dez. 1912)

Einleitung.

Die Erforschung der Viskosität im Zusammenhang mit den Aenderungen der Systembedingungen eines Stoffes ist ein Problem, das in theoretischer Hinsicht von größłter Bedeutung ist. Insbesondere ist es die Frage nach den Beziehungen zum Molekulargewicht kolloider Substanzen, die uns neue Perspektiven zu eröfnen scheint. In dieser Erkenntnis haben in letzter Zeit besonders die Kolloidchemiker angefangen, sich eingehender mit dem Viskositätsproblem $z u$ beschäftigen.

Aber das Problem der Viskosität ist auch für die Praxis von größter Wichtigkeit Bei der Fabrikation der künstlichen Seide handelt es sich darum. immer cine Nitrozellulose von bestimmter Viskosităt zu erhalten. Der Fabrikant muß also die Zügel der Viskosität in seiner Hand haben. Eine genave Kenntnis der Ursachen der so oft $z u$ beobachtenden Viskasitätsänderungen, eine tiefere Einsicht in das Wesen der Viskosität gäbe uns die Mittel in die Hand, die Viskosität jeder beliebigen Nitrozelluloseauflösung nach Belieben zu regulieren.

In der Literatur der Zelluloidindustrie hat man bisher nicht viel von ihr geredet. Allein der Meister weib, daß die Art und Weise, wie sich das Material auf der Walze verarbeiten läb́t, die Elastizităt, die Viskosität der Zellulojdpaste auf die Qualität der fertigen Ware von EinfluB ist. Kolloidchomische Anschauungen haben noch kaum Eingang gefunden in diesen wichtigen Industriezweig. la ihre Bedeutung ist noch nicht einual erkannt und so koinmt es, daß man über die paar inagern Erkenntnisse der molekularen Chemie, die den ganz anders gearteten Verhältnissen nicht Rechnung zu tragen vermag, überhaupt nicht hinauskommen will.

Allgemein verbreitet ist $z . \mathrm{B}$. die Ansicht, dab mit steigendem Stickstoffgehalt der Nitrozellulose auch ihre Viskosität steige. Bis in die neueste Zeit hinein findet man den Satz ausgesprochen, daß die Viskosität der Nitrozellulose eine Funktion ihres N-Gehaltes sei. In dieser Fassung ist der Satz aber sicher falsch. Wer überhaupt einmal quantitaiive Untersuchungen über Viskosităt ausgeführt hat, wird sich bald davon überzeugen können, daß die Viskosität ein viel zu empfindliches Merk- mal ist, um an einem so groben Mabstab abgemessen zu werden. Dies wird im Verlauf dieser Untersuchungen zu größerer Klarheit kommen.

$$
\text { Begriff der Viskosität. }
$$

Ueber den Begriff der Viskosität selbst herrscht zuweilen in technischen Kreisen noch etwas Unklarheit.

Algemein gilt in der Kolloidchemie die Viskosität als Mafistab für die ,innere Reibung“ einer Flüssigkeit. Oft aber sieht man in nichtkolloidchemischen Büchern die Viskosität dem "Flüssigkeitsgrad" einer lösung gleichgestellt. Daraus entspringen zuweilen Irrtümer. Identifiziert man nämlich Viskositä! mit Flussigkeitsgrad, so denkt man unwillkürlich, dał mit der Viskosität auch der Flüssigkeitsgrad einer L.ösung zunimmt.

Das ist aber gerade das Gegenteil von dem. was die Kolloidchemie lehrt, die an der Viskosität die Größe der ,inneren Reibung" einer Lösung abmibt. Diese innere Reibung äubert sich ohne Zweifel in der Arbeit, die anfgewendet werden muB, um die Einzelteilchen gegencinander zu verschieben. Je schwieriger also diese Teilchen sich gegeneinander verschieben lassen, d. h. je woniger flüssig, je zäher eine Lösung ist, desto größer ihre Viskosität:

Man bezeichnet also die Viskosität zweckmäßiger mit ,Zähflüssigkeit" als mit ,Flüssigkeitsgrad" einei Lösung, wenn nan von einem deutschen Worte Gebrauch machen will.

Messung der Viskosität.

Was die Apparate zur Bestinmung der Viskosität betrifft, so scheinen für technische Zwecke das Engler'sche Viskosimeter für dünnflüssige Lösungen, dasjenige von Cocchius für dickflüssige Lösungen am meisten in Gebrauch zu stehen.

Diese Apparate haben aber, wo täglich eine gröbere Zahl von Versuchen auszuführen sind, verschiedene Nachteile, besonders wenn sie für Betriebszwecke in der Zelluloidindustrie zu verwenden sind. Weil sie in solchen Fällen nicht gleichzeitig als Aufbewahrungsgefässe für die zu untersuchenden Lösungen dienen können, die oft tage- oder wochenlang aufzubewahten 
sind, so ist eine grobe Zahl yon Apparaten notwendig, wenn man nicht für jede Bestimmung die Lösung aus- und umgieBen will. Dies erhöht die Kosten natürlich sehr erheblich. Zudem ist die Reinigung immer mit Zeit- und gewissen Materialverlusten verbunden. Auch ist nicht ausgeschlossen, daB das häufige Aus- und Umgieben zu einer Quelle von unerwünschten Veränderungen in der Viskosität werden kann. Ferner sind die Apparate - dem Fabrikchemiker besonders - nicht immer zugänglich.

Auf folgende einfache Weise kann man sich Apparate herstellen, welche diese Uebel nicht besitzen und auch an Genauigkeit obigen Apparaten kaum nachstehen dürften, wenn gewisse MaBregeln nicht vernachlässigt werden.

a) Die Fallmethode: Für dickflüssige Loosungen genügt ein einfacher Glaszylinder, von ca. $30 \mathrm{~cm}$ Höhe mit eingeschlifferen Glasstopfen, durch den noch ein Thermometer eingelassen werden kann. In $3-4 \mathrm{~cm}$ Bodenhöhe kratzt man an der Außenseite des Glases einen Nullstrich ein und $20 \mathrm{~cm}$ höher einen Anfangsstrich. Von der zu untersuchenden Lösung füllt man nun so viel ein, dab sie noch etwa $3 \mathrm{~cm}$ über den Anfangsstrich hinaussteht.

Man bestimmt nun die Zeit, die eine kleine Glaskugel von $7-8 \mathrm{~g}$ braucht, um von dem Anfangsstrich zum Nullstrich zu fallen, d. h. eine Strecke von $20 \mathrm{~cm}$ zurückzulegen.

Der Glaszylinder darf nie ganz volfgefüllt werden, da sonst der Zylinder durch den Druck der sich allfällig unter Wärmeeinflüssen ausdehnenden Flüssigkeit zerspringen kann.

Sollen gut übereinstimmende Werte erhalten werden, so mub die Lösung ganz frei gemacht werden von Schleimklümpchen. Schlieren und Flüssigkeitsfäden dürfen nicht sichtbar sein. Die Lösung muB durchaus homogen ausșehen. Auch Luftblasen sind schädlich. Sic verschwinden gewöhnlich von selbst beim Stehenlassen, während Schleimklümpchen am besten durch kräftiges Rühren zum Verschwinden gebracht werden.

Bei einer Wiederholung des Versuchs wird das Gefäß einfach auf den Kopf gestellt. Die Glaskugel braucht nicht gereinigt zu werden. Der erste Versuch fällt gewöhnlich etwas zu hoch aus, weil am Anfang die Reibung mit der trockenen Glaskugel etwas größer ist, als nachber.

b) Die Auslaufmethode: Für dünnflüssige Lösungen kann man $\mathrm{zu}$ Viskositätsbestimmungen jede Pipette gebrauchen. Man hat dabei nur darauf $z u$ achten, daB man immer bei derselben Temperatur abliest, da mit steigender Temperatur die Viskosität abnimmt. Man bestimmt die Zeit, die zum Auslaufen einer bestimmten Quantität Lósung, z. B. $15 \mathrm{ccm}$, nötig ist. Da diese um so langsamer ausfliebt, je kleiner die Ausfluboffnung, so tut man gut, die Viskosität nicht absolut zu messen, sondern sie als Vergleichszahl anzugeben in bezug auf ein anderes als Einheit festgesetztes Medium. Am besten eignet sich dazu Wasser. Man dividiere also die gefundene Sekundenzahl durch die Ausflußzeit derselben Menge Wassers. Dadurch macht man sich unabhängig von der besonderen $\mathrm{Be}$ schaffenheit der Pipette.

Da gegen das Ende hin eine Flüssigkeit langsamer aus der Pipette flieBt, so verschiebe man den SchluBstrich nach oben und setze ihn einige Zentimeter unter die Ausbuchtung.

Die Pipette muB natürlich senkrecht gehalten werden, und es mub die untere Spitze während des Versuchs etwas in die Flüssigkeit eintauchen, da sonst wegen der Austrocknung der ausfließenden Lösung an der Ausflußöffnung keine übereinstimmenden Resultate erhalten werden können.

Die Lösungen bewahre man am besten in hohen engen Mebzylindern auf, da die Verdunstung in solchen Gefäßen am geringsten ist.

$$
\text { Einflub der Zeit. }
$$

Wird eine Nitrozellulose in Kampferspiritus aufgelöst, so entsteht je nach den Bedingungen eine mehr oder weniger zähe Flüssigkeit, eine kolloide Lösung. Und zwar zeigt sie die typischen Merkmale eines sog. "Emulsionskolloids", schon wegen ihrer groben inneren Reibung. Bei den im Gegensatz dazu stehenden sog. "Suspensionskolloiden" unterscheiden sich die Viskositäten nur wenig von denen ihrer Lösungsmittel. Auch nimmt bei den Emulsionskolloiden die Viskosität ungeheuer rasch $z u$ mit der Konzentration, was auch mit unserer Kampferspirituslösung der Fall ist.

Wir haben damit den Boden der Kolloidchemie betreten, und es sind Erscheinungen zu erwarten, zu denen ein Analogon in der Mole. kularchemie nicht besteht. Dahin gehören die sog. Alterungserscheinungen. Man versteht darunter die Erscheinung, daB beim Stehenlassen, beim Aelterwerden einer Lösung eine $\mathrm{Ab}$ - oder Zunahme der Viskosität eintritt.

So wurden mit einer Kampferalkohollösung einer Nitrozellulose folgende Werte erhalten: 


\begin{tabular}{|c|c|c|c|}
\hline $\begin{array}{c}\text { Als Lösungsmittel } \\
\text { diente } 50 \text { prozentiger Kampleralkoinol }\end{array}$ & $\begin{array}{l}1 \text { prozentige } \\
\text { Lösung }\end{array}$ & $\begin{array}{c}3 \text { prozentige } \\
\text { Lösung }\end{array}$ & Bemerkungen \\
\hline $\begin{array}{l}\text { Die Nitrozellulose bildet nach dem } \\
\text { Lösen eine feste Gallerte, welche } \\
\text { dann mit dem Kampferalkohol so } \\
\text { lange verrährt wird, bis die Flüs- } \\
\text { sigkeit ein ganz homogenes Aus- } \\
\text { sehen bekommt. Alle Klümpchen } \\
\text { müssen sorgfartig aufgelost werden. } \\
\text { Dann erst kann mit der Viskositäts- } \\
\text { bestimmung begonnen werden. } \\
\text { Alle früheren Bestimmungen sind } \\
\text { wertlos. }\end{array}$ & 2,71 & 22,8 & $\begin{array}{c}\text { Nach kürzerer oder längerce Zeit } \\
\text { wird sowohl bei verdünnten wie } \\
\text { bei konzentrierten Lösungen ein } \\
\text { Zustandsverhältnis mäBiger Sta- } \\
\text { bilitat erreicht. }\end{array}$ \\
\hline $\begin{array}{c}\text { Nach } 30 \text { Min. } \\
: 60 \text {. } 1^{1 / 2} " \text { Std. } \\
: 2^{10}: \\
: 13: \\
: 15:\end{array}$ & $\begin{array}{l}2,70 \\
2,77 \\
2,77 \\
2,77 \\
2,86 * \\
2,95 \\
3,14\end{array}$ & $\begin{array}{r}24,66 \\
25,66 \\
27,55 \\
27,63 \\
27,53 \\
27,66 \\
30,55-31 \\
\text { eine lel } \\
\text { eingese } \\
\text { schwan! } \\
\text { seinen } \\
\text { keine }\end{array}$ & $\begin{array}{l}\text { *s ist ersichtlich, daß wieder } \\
\text { taftere VergroBerung der Viskositat } \\
\text { hat. DaB die Werte jetzt stark } \\
\text { n, hat in der Ausflußnethode } \\
\text { und, die für solchedickenLösungen } \\
\text { inheitlichen Werte melr liefert. }\end{array}$ \\
\hline " 14 Tagen & 4,2 & $48-50$ & \\
\hline
\end{tabular}

Wegen der zeitlichen Unbeständigkeit solcher Lösungen ist es auch nicht möglich, von Lösungen derselben Konzentration, derselben Nitrozellulose und desselben Dispersionsmittels stets die gleichen Werte zu erhalten.

Für einprozentige Lösungen sind sie ziemlich gleichmäBig, für stärkere Lösungen aber erhält man um so schwankendere Zahlen, je gröler die Konzentration und die Dickflüssigkeit der Lösung.

Einflub des Lösungsmittels und der Konzentration.

Die Viskosität steigt enorm rasch mit der Konzentration, und zwar so, daB bei Anwendung von 50 prozentigem Kampferalkohol bes über dreiprozentigen Lösungen mit Hilfe der Ausllußmethode schon keine eindeutigen Resultate mehr erhalten werden können.

Was die Alterungserscheinungen betrifft, so sind solche um so ausgeprägter, je konzentrierter die Lösung und je höher die ursprüngliche Zäbflüssigkeit.

0,5 prozentige Kampferalkohol-Nitrozellulosen sind verhältnismäbig viskositätsbeständig und noch dünnere verhalten sich fast wie inolekulare Lösungen.

C. Piest hat zweiprozentige Lösungen von Kollodiunwolle in Azeton und Aetheralkohol während 21 Tagen geprüt und dabei gefunden,

dab sie ihre Viskosität beibehalten, am Ende dieser Zeit eher etwas schwächer werden. Er gibt aber $z u$, daB bei einigen Nitrozellulosen die Viskosität höher wird.

Auch das Lösungsmittel hat einen Einflub auf die Viskositätsgröße und auf deren Beständigkeit.

Nach C. Piest nimmt die Viskositär einer zweiprozentigen Lïsung von stabiler Schiebbaumwolle in Azeton nach 21 Tagen nur wenig ab; eine gleichstarke Lösung einer stabilen Kollodiumwolle in Azeton nimmt etwas stärker $a b$, und dasselbe Produkt in Aetheralkohol wird zuletzt ganz flüssig

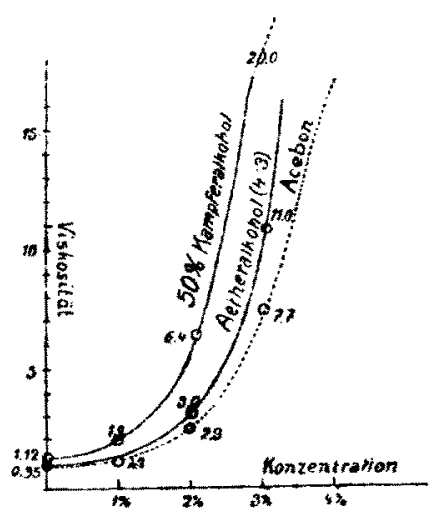

Fig. 1 
Den EinfluB des Lösungsmittels und der Konzentration auf eine Nitrozellulose von 11,05 Proz. $\mathrm{N}$ zeigt die untenstehende Kurve.

Daraus geht also hervor, daB Aetheralkohollösungen stärker viskos sind als Azetonlösungen und dab ferner Kampferalkohollösungen in bezug auf Viskosität die beiden ersten um ein beträchtliches übertreffen.

Kampfergehalt und Viskositat.

Versuche wurden ausgeführt mit 10,40 und 70 prozentigem Kampferalkohol IniterVerwendung ein und derselben Nitrozellulose. Wie die Tabelle zeigt, wurde die Viskosität des Lösungsmittels zuerst für sich bestimmt und täglich je $1 \mathrm{~g}$ Nitrozellulose mehr hinzugefügt, so dab mittags jeweilen die Bestimmungen ausgeführt wurden.

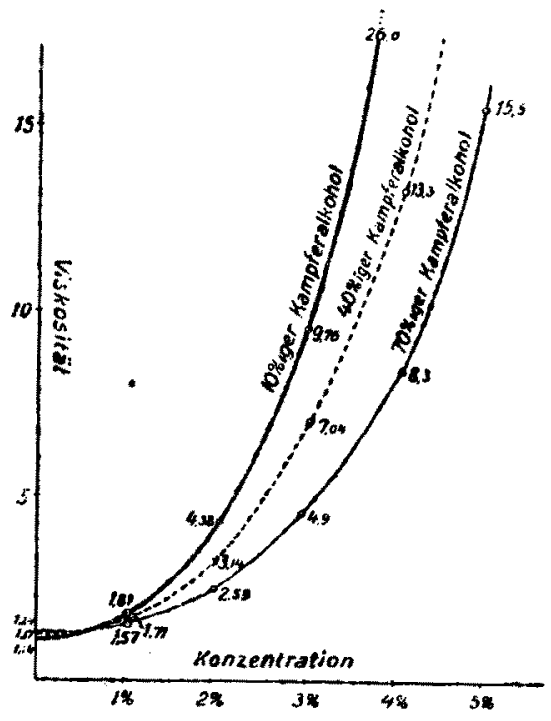

Fig. 2

Die Tabelle zeigt, dal ein hoher Kampfergehalt die Viskosität stark heruntersetzt, was auch mit der Erfahrung übereinstimmt, daB Zelluloid durch hohen Kampierzusatz an Güte gewinnt.

Unangenehm wirken bei solchen Versuchen die Alterungserscheinungen, die bei kampierarmen Lösungen schon bei einer Konzentration von 3 Proz. auftreten können und von da an verzerte Kurven geben mit wertlosen Resultaten.

Auch bei diesen Versuchen zeigte sich, daB die Alterungserscheinungen bei um so höheren Konzentrationen aufzutreten beginnen, je geringer die Viskositäten bei niederen Konzentrationen ausgefallen sind.
Einflub der Temperatur.

Nach verschiedenen vergeblichen Versuchen gelang es mir, nachdem die Lơsung einem Reifungsproze $B$ unterworfen worden war, zuverlăssige Zahlen zu erhalten.

Die Lösung wurde in folgender Weise hergestellt. In einem Gemisch von $100 \mathrm{ccm}$ Alkohol (95 Proz. Vol.) und $60 \mathrm{~g}$ Kampfer wurden $4 \mathrm{~g}$ Kollodiumwolle gelöst, und zwat so, dab jeden Tag je $1 \mathrm{~g}$ davon eingesetzt wurde. Nach Verlauf dieser vier Tage wurde die Lösung weitere sechs Tage sich selbst überlassen. Dieser ReifeprozeB hatte zur Folge, daB die Viskosität ziemlich viel niedriger ausfiel, als wenn die vier Gramme zu gleicher Zeit aufgelost worden wären. Die homogen aussehende Flüssigkeit wurde noch weiter verdünnt mit $50 \mathrm{ccm}$ einer 75 prozentigen Kampferalkohollösung, worauf dann die Viskositäten bestimmt wurden.

Die Lösung wurde auf $60^{\circ} \mathrm{C}$ erwämt und von je $10^{\circ}$ zu $10^{\circ}$ die Viskosität bestimmt. Dann wurde wieder abgekühlt und wiederum von Zeit zu Zeit untersucht.

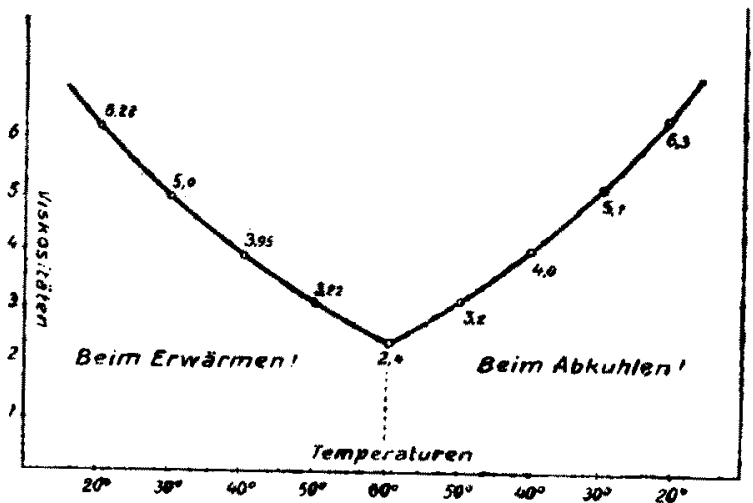

Fig. 3

Werden die erhaltenen Zahlen graphisch aufgezeichnet, so führt die Symmetrie beider Kurven zu dem Schluß, das die durch Temperatureinflüsse entstandenen Verănderungen in der Viskosität reversibler Natun sind.

\section{Einflub des Lichts.}

Darüber war bis jetzt nichts bekannt. Eine einzige Stelle fand ich in der Literatur ${ }^{1}$ ), die darauf Bezug hat. Darnach machte G.W. Mac Donald einst die Beobachtung, daß nach der Behandlung einer Kollodiumwolle mit Aether im Soxhletapparat eine beträchtliche Abnahme der Viskosität eintritt. Als er Viskositätsbestimmungen

1) Journ. Chem. Soc. 13, 785 (1911). 
mit Doppelmustern derselben Kollodiumwolle in Azetonlösung ausführte, erhielt er zu seinem größten Erstaunen ganz verschiedene Zahlen, die unmöglich miteinander zu vergleichen waren. Für die Abnormität seiner Viskositätswerte machte er die Einwirkung des Lichts während der Herstellung solcher Lösungen verantwortlich. Ein zu diesem Zwecke eigens ausgeführtes Experiment soll nach ihm folgendes ergeben haben: "Eine 2,5 prozentige Lösung von Nitrozellulose in Azeton fiel ungefähr auf die Hälfte ihrer anfänglichen Viskosität herunter, nachdem die Lösung sieben Tage lang diffusem Tageslicht ausgesetzt worden war. Kontrollösungen, die einige Wochen lang in Dunkeln standen, zeigen nur eine ganz geringe Abnahme der Viskosität."

G. W. Mac Donald empfahl de Mosenthal in einer Diskussionsangelegenheir über den Einflub des Lichts auf die Viskosität die Frage zu prafen, wenn er in Sinne habe, ein Mittel zu suchen. um die Größe des Moleküls herabzusetzen.

Demgegenüber muB erwidert werden, daB obiger Versuch in mannigfacher Wiederholung mit verschiedenen Kollodiumwollen nicht wieder bestätigt werden konnte. Es ergaben sich bei dieser Konzentration nur geringe Unterschiede, die zum Teil auf Alterungserscheinungen, zutn Teil auf kleine Temperaturunterschiede zurückzuführen waren.

Von Interesse ist ferner eine Bemerkung de Mosenthal's (a.a.O.), dab nach dem Auflosen von Nitrozellulose und ihrer Wiedergewinnung durch Ausfällen oder Eindampien und darauffolgendes Wiederauflösen des Niederschlags oder des Films die Viskosität der Lösung fällt. Und er meint, daB bei mehrmaligem Wiederholen dieses Prozesses Lösungen von sehr geringer Viskosität erhalten werden könnten. "Wenn dies weit genug getrieben wird, so mag es möglich sein, daß eine Grenze des Molekularzustandes gefunden wird, die eine Molekülgewichtsbestimmung nach schon bekannten Methoden ermöglichen wïrde."

Dieser Gedankengang hat sicher etwas Bestehendes und mag sehr wohl geeignet sein, zu solchen Versuchen anzuregen. Allein die vielen Substanzverluste, die notwendig thit diesen Operationen verbunden sind, zusammen init dem Uebelstand, daß all unsere Viskositatsmethoden so grobe Mengen von Substanz benötigen, lassen uns solche Arbeiten als wenig aussichtsvoll erscheinen. Es entsteht vielmehr der Wunsch nach einer Methode, die auch von einer geringen Substanzmenge die Viskosität an- zugeben imstande wäre. Es sei darauf besonders hingewiesen. Vielleicht greift die Mikrochemie diese Anregung auf. Angesichts der großen Bedeutung, die der Viskosität für die Entwicklung kolloidchemischer Anschauungen zukommt, dürfte die Arbeit nicht umsonst sein.

\section{EinfluB von Zusätzen.}

Von der Rolle der Unreinlichkeiten in den Lösungen, von den Wirkungen, die kleine Zusätze auf die Viskosität haben können, ist überhaupt noch nichts in der Literatur zu linden, obschon gelegentlich beobachtet werden kann, daß durch gewisse Zusätze, wie sie in der Fabrikation des Zelluloids zur Neutralisation der letzten Spuren mit oder ohne Erfolg der Zelluloidmasse auf der Walze zugefügt werden, die Sprödigkeit des fertigen Materials unter Umständen erhöht wird.

Ein Laboratoriumsversuch an einer zeitlich stabilen Kampferalkoholauflösung einer Nitrozellulose zeigte mir, dab kleine Zusätze von Harnstofflösung, z. B. im Verhăltnis von ungefähr 1 Proz. zur Lösung addiert, die Viskosität um $10-15$ Proz. erhöhen, während größere Mengen absolut keinerlei Wirkung zeigten, die einzig und allein dem Zusatzmittel hätten zugeschrieben werden müssen. Selbstverständlich werden besondere systematische Untersuchungen nötig sein, um klar zu legen, ob diese kleinen Aenderungen im kolloiden Zustande der Lösung einer katalytischen Wirkung solcher Zusätze zu verdanken sind oder nicht.

Das Studium solcher katalytischen Wirkungen wäre sehr erwünscht, da solche Prozesse in der Chemie des Zelluloids eine grobe Rolle zu spielen scheinen.

\section{Viskosität und Brüchigkeit.}

Beim Zelluloid stehen diese Eigenschaften in einem gewissen Zusammenhang. Offenbar hängt die Brüchigkeit eines Sroffes von seiner inneren Struktur ab. Das geht schon daraus hervor, dab der Kenner an der Beschaffenheit der Bruchfläche die Qualität der Ware abzuschätzen weib und dab eine gute Ware sich durch einen gewissen Glanz ihrer Bruchfläche kennzeichnet. Auch die Tatsache, daB sich nicht alles Zelluloid mit gleicher Leichtigkeit zu Hochglanx polieren $1 \mathrm{~F} B \mathrm{Bt}$, ist wohl auf Verschiedenheit in der Struktur des Materials und auf die Beschaffenheit ihrer inneren Oberfläche zurückzuführen.

Damit schon dürite wohl klar geworden sein, von wie grobem Interesse ultramikro- 
skopische Untersuchungen von Zelluloid sein würden. Sie würden vielleicht imstande sein, uns Aufschluß zu geben über die strukturelle Beschaffenheit dieser Stoffe und uns über den kolloidchemischen Werdeproze $B$ des Zelluloids aufzuklären, der doch nur als ein UmwnndlungsprozeB eines Systems von der Zusammensetzung $\mathbf{F}+\mathbf{F}^{2}$ ) über den Typus $\mathrm{FI}+\mathrm{Fl}^{3}$ ) in das Endsystem der Zusammensetzung $F+F^{4}$ ) aufzufassen ist.

Offenbar hat die Braichigkeit für einen festen Stoff ungefähr das zu bedeuten. was die Viskosität für einen flüssigen Körper ist. Beide Begriffe geben schlieBlich die Kräte an, mit welchen die einzelnen Teilchen eines festen oder flüssigen Stoffes aneinander haften oder sich gegeneinander verschieben lassen.

Immerhin ist die Sache nicht so einfach. daß man einınal denken könnte, durch ein einfaches Zahlenverhältnis diese beiden Eigenschaften in Zusammenhang zu bringen und das man z. B. sagen honnte, wenn die Viskosităt in dem und dem Zustande der Fabrikation soundso viel beträgt, die Elastizität des Stoffes die gewünschte sein wird. Und zwar um so weniger, als wir für die Viskosität, wie sie für das Material beim Verwalzen in Betracht kommt, keine Methode haben zu quantitativer Bestimmung. Infolge der großen Zähigkeit versagen selbstverständlich sowohl Auslaufmethode wie auch Fallmethode. Nicht die feinen Methoden des Chemikers geben also Antwort auf die Frage nach der voraussichtlichen Beschaffenheit der Ware in bezug auf Elastizität, Brüchigkeit, Glanzfähigkeit, Stabilität usw., sondern die grobe Hand des Meisters löst dieses Problem, indem sie sich ein Stück von der Walze reiBt, und es auf seine Ausstreckfăhigkeit hin prüft. Ein sog. „kurzes Material“ wird nicht gerne gesehen. Es gilt von schlimmer Vorbedeutung für die physikalischen Eigenschaften des fertigen Materials.

Es ist $z u$ bedauern, dab wir auch keine zahlenmäßigen Relationen haben zwischen der Festigkeit oder Zähigkeit des teigähnlichen Materials und der Viskosität desselben Materials in ganz flüssigem Zustand. So viel kann nur gesagt werden, daß Brüchigkeit des fertigen Materials, die Elastizität des Gels in halbfertigem Zustand und die Viskosität der dünnflüs: Nitrozelluloseauflösung in Kampferalkohol, wie sie bei Beginn der Verarbeitung der Nitrozellulose

3) Kampferalkohol + Nitrozellulose.

7) Zelluloidgel.

9 Fertiges Zelluloid. im Laboratorium versucht zu werden pflegen, in einem gewissen Verhaltnis stehen und daB man in der Praxis die Erfahrung macht, das eine gut getrocknete Kollodiumwolle im Gewichtsverhältnis von $1: 12,5$ in 75 prozentigem Kampferalkohol anfgelöst auf der Walze ein um so besseres Resultat gibt, je dünnflüssiger diese Lösung ist, d. h. je kleiner ihre Viskositat. Allerdings nur unter einer Voraussetzung kann ein solcher Versuch für den Betrieb von Nutzen sein, nămlich nur dann, wenn von dem Augenblick an, wo die vollig fertige Nitrozellulose zu Zelluloid verarbeitet ru werden beginnt, bis zu dem Moment an, wo sie auf die Walze kommt, alle tuBeren Einflüsse, welche die Viskosität dauernd zu verändern vermbichten, mit aller Sorgfalt und Vorsicht auszuschlieBen gesucht werden. Nur unter diesen Bedingungen vermag uns eine solche vorlaufige Prufung in Laboratorium bei etwaigen Fehlresultaten im Betrieb vor fehlerhaften Schlilssen zu bewahren.

\section{Natur der Nitrozellulose und Viskositut.}

Wenn wir uns von Kollodiumwollen annähernd gleichen $\mathrm{N}$-Gehalts, Kollodiumwollen, die $z u$ verschiedenen Zeiten, aber unter sonst ganz gleichen Bedingungen hergestellt wurden, wenn wir uns von solchen Kollodiumwollen z. B. zweiprozentige Kampferalkohol-oder Azetonlösungen bereiten, so zeigen sie in bezug auf Viskosităt oft erstaunliche Unterschiede, die auf keine der bisher betrachteten Einflüsse zurückzuführen sind, sondern deren Grund sicherlich in der Natur der erhaltenen Nitrozellulosen selbst liegt.

Durchmustern wir einmal alle jene Paktoren, die beim Nitrieren, beim Waschen und Bleichen der Nitrozellulose diese in ihrer Natur, wie sie sich besonders in den Viskositätswerten ăßBert, beeinflussen können, soweit sie bis jetzt schon untersucht worden sind.

\section{Der NitrierungsprozeB.}

a) Temperatur des Bades: Je höher die Temperatur des Bades bei sonst gleichen Bedingungen, umso höher der N-Gehalt der Kollodiumwolle, umso höher ihre Löslichkeit und desto niedriger die Viskosität und die Ausbeuten. (C. Piest a. a. O.)

b) Wassergehalt des Bades: Je konzentrierter das Bad, d. h. je weniger Wasser das Bad enthalt, umso höher der N-Gehalt und umso hôher die Viskosität. (Berl und Klaye.) 
c) Nitrierungsgrad: Die Viskositătvon Nitrozelluloselösungen in Azeton wächst - bei gleicher Nitrierungsdauer und gleicher Nitriertemperatur - mit Steigerung des N-Gehalts. (C. Piest.)

d) Nitrierungsdauer: Mit der Ninitrungsdauer nimmt im allgemeinen unter sonst gleichen Bedingungen auch die Viskosität der Lösung ab. (G. Lunge.)

G. Lunge sprach die Ansicht aus, dab wohl eine Depolymerisation des Moleküls stattinde und vielleicht eine Bildung von Hydro- oder Oxyzelluloseäther einsetze.

Zur weiteren Prüfung dieser Frage versuchte ich von Proben, die von fünf $z u$ fünf Minuten einem groBen Nitrierungsstoff entnommen wurden, Stickstoffgehalt und Viskosität zu bestimmen. Das letztere geschah nach der Fallmethode, da die Nitrozellulose im Verhältnis von $1: 12,5$ in 50 prozentigem Kampferalkohol aufgelöst worden war.

Auf die untenstehenden Resultate werde ich später wieder zurückkommen.

\begin{tabular}{|c|c|c|c|}
\hline Nitrietzeit & N-Proz.: Proz. & $\begin{array}{l}\text { Viskosität } \\
\text { Sekunden }\end{array}$ & Bemerkungen \\
\hline $\begin{array}{l}5 \text { Minuten } \\
10 \\
15 \\
20 \\
25 \\
30 \\
35 \\
40: \\
45: \\
50: \\
55: \\
60 \\
11 / 2 \\
6 \\
24\end{array}$ & $\begin{array}{r}8,97 \\
10,05 \\
10,39 \\
10,45 \\
10,74 \\
10,75 \\
10,81 \\
10,84 \\
10,85 \\
10,90 \\
10,92 \\
10,99 \\
10,93 \\
11,09 \\
11,44\end{array}$ & $\begin{array}{l}\left.-a^{4}\right) \\
\left.29^{3}\right) \\
\left.34^{8}\right) \\
\left.14^{4}\right) \\
\left.26^{5}\right) \\
14 \\
20 \\
13 \\
18 \\
16 \\
12 \\
13 \\
12 \\
8 \\
6\end{array}$ & 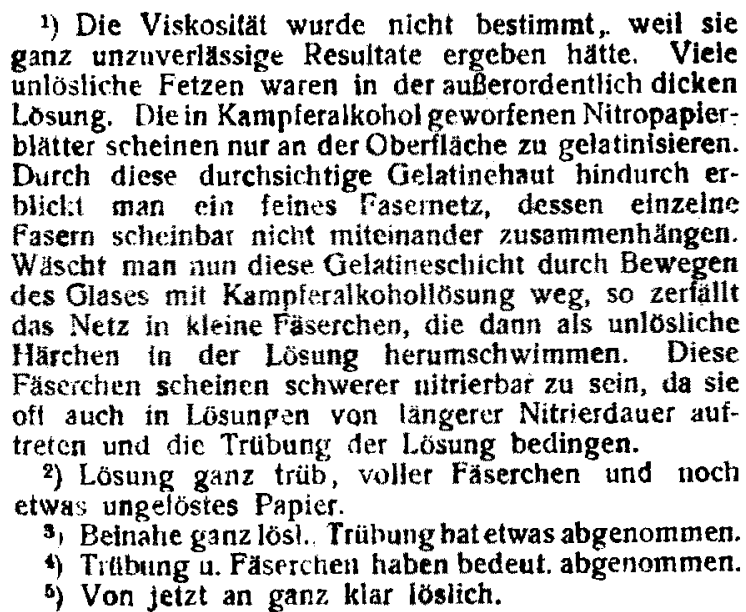 \\
\hline
\end{tabular}

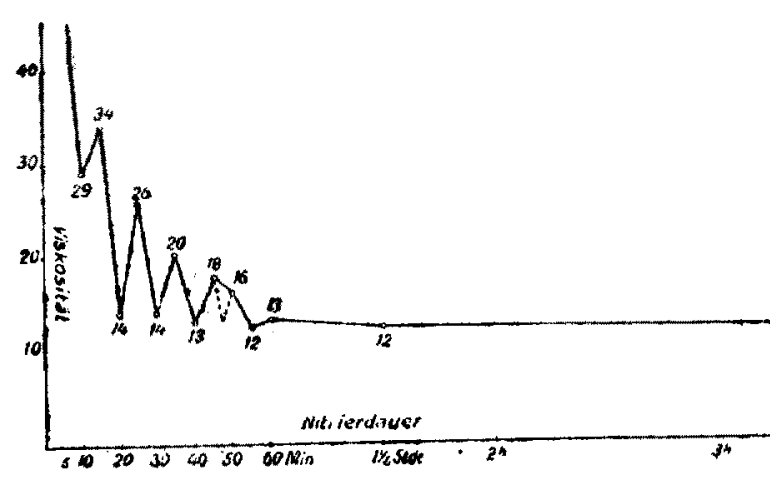

Fig. 4

2. Der Waschprozeb.

Spuren von Säuren, die im Zelluloid zurückbleiben, können von ungenügendem Waschen oder von den Unreinichkeiten in den industriellen 1.ösungsmitteln herrühren. Bezüglich des Waschens gehen die Meinungen zur Zeit stark auseinander.
Nach G. l.unge erniedrigen Spuren von Säuren die Viskosität der Nitrozellulose für Aetheralkohollosungen. Er meint aber, dal ganz kleine Mengen von Säuren keinen großen Einfluk haber.

Demgegenüber behauptet $\left.C \cdot P_{i} \in t^{5}\right)$ dat zweiprozentige Lösungen sowohl stabiler wie unstabiler Kollodiumwolle in Azeton oder Aetheralkohol ihre Viskosität überhaupt nicht vermindern, und zwar gleichgültig, ob sie schwach angesäuert werden oder nicht. Er hat die Versuche 21 Tage iang fortgesetzt und bei einigen Nitrozellulosen sogar eine Erhöhung der Viskosität konstatieren lönnen.

Dab G. Lunge Veränderungen in der Viskositäl vorfand, ist nach $C$. Pie st dem groben Zusatz von Săure zuzuschreiben. G. Lunge verweridete auf $100 \mathrm{~g}$ Lösung $1 \mathrm{~g}$ Wasser, $0,25 \mathrm{~g}$ Schwefelsăure und $0,25 \mathrm{~g}$ Salpetersäure.

5) Zeitschr. f. ges. Schieb- und Sprengstoff-Wesen vom 1. Nov. 1910. 
Neuere Betriebserfahrungen haben aber gezeigt, dab Spuren von Säuren doch von grobem EinfluB auf die Viskosität der Nitrozellulosen sein können.

Wichtig ist ferner die Tatsache, dabs eine große Zahl von Waschungen kurzer Dauer der Stabilisation in hohem MaBe günstig sind und dath sie die Viskosität der Nitrozellulose-Auflösungen viel stärker vermindern als eine kleine Zahl von Waschungen von langer Dauer.

Eigentümlich ist ferner die von $C$. Piest erwahnte Beobachtung, dab die letzte Waschung der stabilen Nitrozellulose eine wesentliche Verminderung der Viskosität hervorruft. Dasselbe wird bemerkt bei unstabilen Nitrozellulosen, welche gerade nach dem Nitrieren einige Waschungen erfahren haben.

\section{Der BleichprozeB.}

Stark gebleichte Baumwollen geben gewöhnlich niedrige Viskositätswerte.

Damit stimmt auch dic von Berl und Klaye gemachte Erfahrung iiberein, dab unter denselben Nirierbedingungen Azetonlösungen reiner Nitrozellulosen viel höhere Vis. kositätswerte aufweisen als die Nitroverbindungen der Hydro- oder Oxyzellulose, mit denen gebleichte Stoffe angereichert sind.

Damit hängt natürlich auch die Viskorität von der ursprïnglichen Beschaftenheit des Rohmaterials $a b$ und von dessen chemischen Verảnderungen während des der Nitrierung vorausgehenden Trocknungsprozesses.

\section{Ueber das Molekulargewicht der Zellulose.}

Es hat fast den Anschein, als ob die Erforschung des Zellulosemoleküls an einem toten Punkt angelangt sei. Es scheint, als wenn wichrige theoretische Fortschritte nur für jene Zeit in Aussicht stehen, in der die Frage nach dem Molekulargewicht der Zellulose eindeutig gelöst sein werde; denn bekanntlich herrscht in dieser wichtigen Frage sehr wenig Uebereinstimmung.

A. G. Green ist der Ansicht, daB die Cründe, welche die Veranlassung sind, für die Zellulose ein Multiplum der Formel $\mathrm{C}_{6} \mathrm{H}_{10} \mathrm{O}_{5}$ anzunehmen, nicht genügend gestützt sind. Und bevor nicht weitere Beweise für ein höheres Molekulargewicht gegeben sind, glaubt er, daB der Zellulose die einfache Formel $\mathrm{C}_{6} \mathrm{H}_{10} \mathrm{O}_{5}$ zuzuschreiben sei, die er gleichzeitig als ein inneres Anhydrid der Glukose darstellt ${ }^{6}$ ):<smiles></smiles>

Nastuk off gelangte durch seine Untersuchungen ${ }^{7}$ ) $z u$ der Ueberzeugung, dab das Molekulargewicht der Zellulose das vierfache sei. G. Lunge und Bebie hatten diese Formel auf Grund ihrer Nitrierungsversuche immer für die richtige gehalten.

Bumcke und Wolffenstein fanden aus dem Molekulargewicht von Nitrohydrozellulose als Minimum die Zahl 12. Demnach entspricht die MolekulargröBe der Zellulose zum mindesten der Formel $\mathrm{C}_{72} \mathrm{H}_{120} \mathrm{O}_{60}$. Zd. S k r a up berechnete sogar das 34 fache $^{9}$ ).

Es scheint also, dab man von der Lösung dieses Problems weiter entfernt sei, denn je. Und wirklich kommt denn auch Grandmougin (16) zu der Erklärung, dab eine Entscheidung, ob nur ein einfaches oder ein 4, ein 12, ein 34 faches Molekulargewicht bei der Zellulose vorliege, zur Zeit ein Ding der Unmöglichkeit sei.

Man geht dabei stillschweigend von der Voraussetzung aus, daB das Molekulargewicht eines chemischen Stoffes inmer eine konstante GröRe sei und daB auch die Zellulose nur eine MolekulargewichtsgröRe haben könne. Die Chemie har diese Voraussetzung ja an tausenden von Substanzen bisher so oft bestatigt.

Ich bin nicht in der Lage, die bis dahin ausgefinhrten Molekulargewichts-Bestimmungen einer kritischen Prüfung zu unterziehen. Es besteht aber kein Zweifel darüber, daB die an diesem Problem beteiligten Forscher sich der Tragweite und der Wichtigkeit dieses Problems und ihrer Schlubfolgerungen bewubt waren und $\mathrm{da} B$ sie es weder an Vorsicht noch an Bedachtsamkeit fehlen ließen, um zu den zuverlässigsten Resultaten 20 gelangen. Auch ist daran nicht zu zweifeln, dab sie es gewagt hätten, die Richtigkeit ihrer Ergebnisse selbst in Zweiiel zu setzen, wenn sie ihrerseits dazu itgendwclche Veranlassung gefunden hätten.

\footnotetext{
9) Zeitschr. f. angew. Chem. 1904, 1121.

7) Ber. 1900,224 !

g) Ber. $1899,2493$.

9) Wiener Monatshefte 1906,1415 .

19) Chem.-Ztg. 1908, 242.
} 
Man ist sich dessen völlig bewubt und man neigt deshalb dazu, nicht die Forscher selbst, sondern die ihnen zur Verfügung stehenden Methoden verantwortlich zu machen. Allein mir will es scheinen, dak man wohl mindestens ebenso berechtigt wäre, aus der Tatsache, daß die Molekulargewichtsbestimmungen keine übereinstimmenden Resultate liefern, cinen positiven SchluB zu ziehen, siatt dieses negativen, und dab man sich ernetlich einmal die Frage vorlegen sollte, ob es denn wirktich ganz ausgeschlossen sei, dat das Molckiil der Zellulose, das unter gewissen Bedingungen mit dem vierfachen Molekülgewicht auftritt, unter anderen Umständen nicht vielleicht auch wieder als ein Komplex von 2 wölf oder mely Molekitien erscheinen könne? $\mathrm{lch}$ hege dis Ueberzeugung, daB diese Annahme für das Máeliül der Zellulose mehr Berechtigung hat, als die allgemeiner verbreitete daB die Molekülgewichtsbestimmungen sant ihren Methoden nichls wert seien, weil sie keine ibereinstimmenden Resultate geben.

Diese Anschauung von dem wechselnden Molekulargewicht bielet dun 7 eniker, der init den Erscheinungen der Kulluiuchemie etwas vertrauter ist, ïberhaupt schon nichts Neues mehr dar. Es urr uns solche Vorstellungen schon zur Genüge ints $d t w$ anorganischen Chemie bekannt.

Wir haben z. B. das Eisenoxydhydrat. In gewöhnlichem Zustand ist sein Molekulargewicht, wie es seiner Konstitution entspricht, nämlich $\mathrm{Fe}_{2}(\mathrm{OH})_{6}=213,8$. In kolloider Lösung aber kann das, was wir als Molekuilgewicht ermitteln, hinaufgehen his zu 6000 . Das. was hier als Einheit zusammenhalt, kamn nicht mehr das isolierte Molekül sein, es mub eine Gruppe von Molekülen sein, eine höhere Einheit.

Ein weiterer Grund für die Annahne einer weitgehenden Polymerisation des Zellulosemoleküls liegt in seiner Fähigkeit, Fasern zu bilden. Dadurch schon unterscheidet sich die: Zellulose von allen anderen chcmischeu Stoffen In analoger Weise, wie mall den Bau von Kristallen auf molekulare Verhäitnisse zurückführt, so versucht man den Bau der Faser und ihrer Leistungen auf solche der sie zusammensetzenden Zellulosenmoleküle $z u$ gründen. Dies dürte aber eben so wenig gelingen, wie es unmöglich ist, ein Haus aus bloken Sandkötnern aufzubauen, obschon man diese als seine Urelemente betrachten muB. Wie diese sich zu gröBeren Einheiten, zu festem Baumaterial verdichten lassen müssen, so muß das Zellulosemolekül mit jener
Fähigkeit begabt sein, höhere Einheiten zu bilden; Komplexe, die sich schlieblich zu dem so komplizierten Bau der "Paser" zusammenfügen.

Die Baumwollfaser ist aber auch der Träger physiologischer Funktionen; denn in ihr spielen sich Lebensprozesse $a b$. Und für diesen ist es charakteristisch, daB in ihm mit dem Vorgang der Zerstörung derjenige der Bildung verbunden ist. Aus diesem Grunde schon haben isolierte, starre, echtgelöste Moleküle nichts mehr, was dem Leben änlich ist. Denn diese sind im Zustande der Stabilitat. Sie sind stabil in Form und Grobe, stabil in ihren Leistungen, stabil in bezug auf ihre Zukunft, stabil auf ihre Vergangenheit. Nur dadurch, dab man das Molekül der Zellulose nicht als etwas Isoliertes. etwas Bleibendes sich denkt, nur dadurch, daB man sich dasselbe als etwas mit der Fähigkeit Beyabtes sich vorstellt, zil komplexen Gruppen sich zu vereinigen und wieder in solche zu zerfallen, kann eine Annăherung an denjenigen Zustand der Materie zustande kommen, der physiologis he leisłungsfähigkeit in sich schliebt.

Es sei mar auch die Prage gestattet: Liegt nicht vielleicht schon in den vereinzelten und gelegentlich gefundenen Zahlenwerten für den Koeffizieriten des Zellulosemoleküls, in den Zahlen 1, 4. 12, 34 der Keim für eine noch verhüllte Gesetzmäßigkeit, die anzudeuten vermöchte, nach welchen Regeln sich diese Komplexbildung vollzieht? Liegt nicht die Chemie des Zellulosemoleküls vielleicht an demselben Punkte ihrer Entwicklung still, wie die anorganische Chemie zur Zeit, als sie mit den Verbindungen gesättigter Moleküle unter sich, den sogen. Molekülverbindungen, nichts anzufangen wubte? Wieviel Neues und wieviel Licht hat nicht ein vorutteilfreies Studium in diesen Gebieten zulage gefördert? $\mathrm{Ob}$ wir nicht hier etwas Aehniches zu erwarten haben?!

Sei dem, wie ihm wolle. Klar ist nur, dab eine Revision und Fortsetzung der Molekülgewichtsbestimmungen und eine erneute Prüfung der ihnen zugrunde liegenden Voraussetzungen vom kolloidchemischen Standpunkt aus, den Fortschritten der Zellulosechemie jedenfalls förderlicher sein wird, als der Hinweis auf die Aussichts- und Nutzlosigkeit solcher Untersuchungen. Ferner zeigt die Tatsache, daß die Zellulose bei großer chemischer Trägheit doch in physikalischer Hinsicht so viel Mannigfaltigkeit zeigt und zu physiologischer Arbeit befähigt ist, deutlich genug, daß sich gerade die jüngsten Schwestern unserer Wissenschaft, die physikalische Chemie, Ultramikroskopie und Kolloid- 
chemie, miteinander vereinigen müssen, wenn sie den Schleier lüften wollen, der über den Geheimnissen der Zellulosechemie liegt.

Molekulargewicht und Viskosität.

Was die neuen Molekulargewichts-Methoden betrifft, an die man dabei etwa denken mag, so ist es erfreulich $z u$ hören, dab man die Viskosität damit in Bezichung $z \mathfrak{u}$ bringen hofft. Wo. Ostwald hat ja bereits ausgeführt, dat mit steigendem Molekulargewicht die innere Reibung zu wachsen scheint.

Berl und Bütler haben - vielleicht zum ersten Mal - bei ihren praktischen Arbeiten einen darauf sich gründenden Schlub gezogen, als sie die Viskosität von Stärke- und Zellulosenitratlosungen untersuchten. Die Ausflubreit für eine fünfprozentige Nitrozellulose war $9000 \mathrm{mal}$ so groß wie die der gleich konzentrierten Stärkenitratlösung, und daraus nun schlossen die beiden Autoren auf ein wesentlich höheres Molekulargewicht für die Zellulose.

Die beiden Resultate lassen sich aber schon deshalb nicht initeinander vergleichen, weil bei solch hoher Konzentration der Zustand der dispersen Plase viel zu weit entfernt ist von demjenigen der molekularen Verteilung und deshalb auch Alterungserscheinungen und molekulare Polymerisationen bei beiden Substanzen in ganz verschiedenem MaBe zur Geltung kommen werden, weshalb also die Schiubfolgerung Berl's durchaus nicht als eirwandfrei angesehen werden darf Für minimale Molekulargewichtsbestimmunge dürfen von kolloiden Lösungen jedenfalls nur solche von ganz großer Verdünnung in Betrachi gezogen werden; denn der Zustand der molekularen Dispersion einer Lơsung ist dafür ausschlaggebend.

Wenn wir die Viskosität weilerhin in ihrer Abhängigkeit von dem Molekulargewicht einer Substanz betrachten wollen, so mag eine Tatsache besprochen werden, die noch beredter zugunsten einer Polymerisationsfähigkeit des Zellulosemoleküls spricht, als alle bisher aufgeführten Gründe. Es ist dies der merkwürdig rasche Wechsel der Viskositäten während der Nitrierung der Zellulose. Da wir uns etwas eingehender mit dieser Erscheinung zu befassen gedenken, so verdienen die auf Seite 38 wiedergegebenen Werte in graphischer Form noch einmal übersichtlich dargestell z.u werden (siehe Pigur 4, S. 38).

Diese Kurve bestätigt nicht nur die allgemeine, schon erwähnte Regel, dab mit der Nitrierzeit auch unter sonst gleichen Bedingungen und für ein bestimmtes Bad die Viskosităt ab- nimmt, sondern sie zeigt auch, dah sie bis zum Zeitpunkt, wo die Stickstoffaufnahme abzulaufen beginnt, die gröbten Schwankungen zeigt.

Es muB zugegeben werden, daB wegen der Verwendung von Kampferalkohol als Lösungsmittel die Durchsichtigkeit und die Eindeutig. keit der in Betracht kommenden Erscheinungen etwas Schaden leiden. Allein der Umstand, dab die Kurve eine so typische ist und bei jeder Wiederholung des Experimentes dieselbe Gestalt wieder annimmt, läbt wohl den Schlub $z u$, dab die Viskosität auf den speziellen Charakter der jeweilig entstehenden Nitrozellulose zurückzuführen ist und dab uns diese Kurve gewisse Vorgänge während der Nitrierung enthuillt, die in einem vielleicht periodisch sich vollziehenden Auf- und Abbau der Einzelteilchen ihren Grund haben.

Man sieht: Die normalen Viskositätswerte werden erst nach langerer Nitrierzeit erreicht und vorher schwankt die Viskosität von übernormalen zu unternormalen Werten. Dies erklärt auch die beim Nitrieren gemachte Erfahrung, daß eine langere Nitrierung zur Zeit von einer oder anderthalb Stunden zu einem viel gleichmäBigeren Produkte führt als bei nur halbstündiger Nitrierungsdauer, selbst wenn die Produkte innerhalb ihrer Nitrierstufe in bezug auf ihren $\mathrm{N}$-Gehait konstant bleiben.

Offenbar ist das Nitrierbad von zweifacher Wirkung auf den Zellulosekomplex: Einerseits aufbauend, andererseits abbauend.

Erhöhend auf das Molekulargewicht wirkt das Bad offenbar schon durch seinen Ersatz von $\mathrm{OH}$-Gruppen durch $\mathrm{NO}_{3}$, und zwar so, daB beim Ansteigen des $\mathrm{N}$-Gehalts in den ersten fünf Minuten das Molekulargewicht der Zellulose für Kollodiumwollen schon um mehr als die Hälfte zugenommen hat. Darauf folgt ein Sturz in der Viskosität, vielleicht ein Zusammenbruch des Komplexes, durch den wohl der Nitrierung weitere $\mathrm{OH}-\mathrm{Gruppen}$ erschlossen werden, was die Molekulargewichte wieder anwachsen lassen mag usw.

Die abbauende Wirkung des Bades könnte auch mit seinem Wassergehalt in Zusammenhang stehen. In diesem Fall müBte aber jedenfâlls der Tatsache Rechnung getragen werden, daß je grober diese Hydratisierung wird, um so kleiner die Aufnahmefähigkeitt für die $\mathrm{NO}_{3}$-Gruppen.

Wenn diese Scḩwankungen auf Polymerisation und Depolymerisation der Nitrozellulosemoleküle beruhen, so mußs es unter den noch nicht zutn Gleichgewichr gekommenen Nitro- 
zellulosen solche geben, deren Moleküle die Tendenz zum Wachsen haben neben anderen, deren Moleküle zu einer Verkleinerung neigen. Wird die Nitrozellulose getrocknet, so werden solche Vorgänge wohl zum Stillstand kommen. Wenn aber die Nitrozellulose wieder aufgelöst wird, z. B. in Kampferalkohol, so beobachtet man häufig Lösungen, deren Viskosität beim Altern abnimnt, während andere Lösungen mit der Zeit dickflüssiger werden. Diese eigentünliche Tatsache liebe sich leicht dadurch erklären, daß man es im ersten Fall mit Nitrozellulosen $z u$ tun hätte, deren Komplexe $\% u$ einer Vergrößerung neigen, während im zweiten Falle die Komplexe zu einer Verkleinerung tendieren, also offenbar mit Nitrozellilosen, die ihren Gleichgewichtszustand in bezug auf ihre Molekulargröße während der Nitrierung noch nicht erreicht hatten, die also sozusagen $z u$ frühe dem Bad entnommen waren.

Davon nur ein Beispiel. Von zwei Nitrozellulosemustern, die zu gleicher Zeit in demselben Bad nitriert und in gleicher Konzentration in Kampferalkohol aufgelöst worden waren, erhielt ich die untenstehenden Resultate. Der einzige Unterschied war der, daf das eine Muster 30 Minuten, das andere 35 Minuten im Bad gelegen hatte.

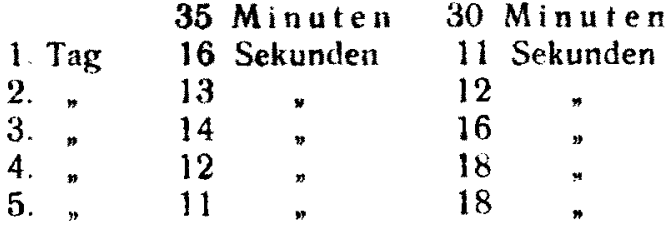

Die Versuche wurden zu gleicher Tagesstunde ausgeführt, mit jeweiliger Unterbrechung von 24 Stunden. Die obigen Werte stellen die Durchschnittswerte dar von drei bis fünf Versuchen, die jeweilig gut miteinander übereinstimmten.

Die Viskosität ist also in einem Fall gestiegen, im anderen Fall gesunken!

Es verdient besonders hervorgehoben zu werden, daB die zahlreichen Viskositätsversuche, die ich an einstïndig nitrierten Nitrozellulosen auszuführen Gelegenheit hatte, fast ausnahmslos wach s e nd e Alterungswerte ergaben, während kurze Zeit nitrierte Zellulosen in ganz unregelmäBiger Weise bald steigende, bald fallende Werte zeigten.

Es wird natürlich noch vieler Experimente bedürfen, um Sicheres über diese Komplexbildungungen in Lösuugen zu erfahren. Ich bin mir der Unvollkommenheit dieser Untersuchungen vollkommen bewubt. Allein es ist mir nicht möglich, die Sache selbst weiter zu verfolgen. Das ist der Grund, warum ich meine Erfahrungen der Oeffentlichkeit übergebe. Es geschieh in der Einsicht, daß eine Größe, die schon bei den unmerklichsten Aenderungen der Systembildungen mefbare Werte liefert, uns umgekehrt auch dazu wird dienen können, molekulare Veränderungen im Innern eines Mediums aufzuhellen, wo uns alle anderen Hilfsmittel im Stiche lassen. So erscheint uns die Viskosität gewissermaBen als ein VergröBerungsglas, mit welchem wir es vielleicht einmal lernen werden, das geheimnisvolle Dunkel des kolloiden $\mathrm{Zu}$. standes $z u$ einigem Licht $z u$ bringen. Hat doch schon der Klassiker Th. Grah am das Viskosimeter geradezu als ein "Kolloidoskop" bezeichnet

\title{
Kolloidchemie und Photographie.
}

\author{
Von Lüppo-Cramer.
}

(Eingegangen am 12. Dezember 1912)

(Wissenschaftl. Laboratorium der Dr. C. Schleu日ner-A.-G. in Frankfurt a. M.)

XVIII. Ueber die quantitativen und topographischen Verhaltnisse beim latenten Bilde und die Reaktionen der Keimbloßlegung.

Der vom Verfasser vertretenen Auffassung der Photohaloide und des latenten Bildes als Adsorptionsverbindungen von Halogensilber mit Silber sind inzwischen bereits zahlreiche Forscher beigetreten. Außer R.Lorenz'), der schon vor mir

1) R. Lorenz, Elektrolyse geschmolzener Salze, II. (Halle 1905), 16. R. A begg's Silberkeimtheorie (Arch. wiss. Phot. 1, 16) kann nicht eigentlich als Adsorptionstheorie mitgezählt werden, da R. A begg Adsorptionserscheinungen nicht in Betracht zog. jene Annahme vermutungsweise aussprach, sind hier von neweren Autoren zu nennen: P. P von Weimarn ${ }^{2}$, W. Reinders ${ }^{3}$, R. Ed.Liesegang ${ }^{4}$, R.Zsig mondy ${ }^{5}$ ), W.D.Bancrof $t^{6}$ ). Auch eine Bemerkung von Wo. Ostwald ${ }^{7}$ ) über

9) Koll.-Zeitschr. 5, 66 (1909).

8) Koll.-Zeitschr. 9, 10 (1911).

4) Koll.-Zeitsctr. 9, 290 (1911).

5) R. Zsigmondy, Kolloidchemie (Leipzig 1912), 202.

b) VIII. Int. Kongr. f. angew. Chemie. Original Communications 20,51; auch Brit. Journ. of Phot. $1912,51$.

7) Koll. Zeitschs. 11, 206 (1912). 